\title{
PLS-NEURAL NETWORK MODEL FOR STOCK PRICES PREDICTION
}

\author{
Meenakshi Sundaram S. ${ }^{1}$, Lakshmi M. $^{2}$ \\ ${ }^{1}$ Research Scholar, ${ }^{2}$ Professor and Head \\ Faculty of Computer Science and Engineering, Sathyabama University, Tamilnadu, India \\ Email: sundarambhu@ rediffmail.com
}

\begin{abstract}
Stock index data are highly volatile in nature and changes over a period of time. Prediction of these are really challenging and computational models are very useful. A computational model called PLS-Neural network has been used in this study. To explore stock market tendency the closing prices with thirteen variables are considered from te BSE sensex data. To evaluate the prediction ability of the models, standard error values are calculated. PLS regression together with Neural network gave a good prediction using a common error measure.
\end{abstract}

Keywords Prediction, RMSE, ANN, Volatility, Regression.

\section{INTRODUCTION}

Interest in financial markets has increased in the last couple of decade, among participants, private-sector market operators, policymakers and academics. Forecasting financial market (stock prices and index etc) have been regarded as one of the most challenging applications and is studied by researchers and practitioners extensively. It is often argued that financial market is very efficient. Therefore, it is difficult to make short term and long term forecasting efficiently. A large body of literature has been accumulated over many years concerning the validity of the efficient market hypothesis (EMH) with respect to stock markets. Predicting the stock market is very difficult since it depends on several known and unknown factors. The power of neural networks is its ability to model a nonlinear process without a priori knowledge about the nature of the process. In this paper we have made an attempt to explore the emerging field of artificial neural network to the complex task of modeling stock prices in the Indian context.

Stock Index prediction has gained importance in recent times due to its commercial application and the nature of the data explored. The noisy environment of the data attracts many researchers to explore it. Many technical indicators are available to predict the daily prices. Each index has its own meaning and interpretation. In this study the indicators considered were daily opening price, high price, low price, volume of transaction, Adjusted Closing prices, Stochastic Oscillator, Rate of convergence, Moving averages, disparity and momentum for predicting the closing prices from $1^{\text {st }}$ January 2008 till $31^{\text {st }}$ December 2010(737).

All traditional parametric time series models like ARIMA, GARCH are valid only when the normality assumption is itself justified. Violation of normality results in using the Nonparametric Models as the only alternative. Partial Least Square regression, Principal Component Analysis, Ridge Regression models are some of the Classical Statistical Models and Neural Network is the most widely used computational non parametric model. Artificial Neural Network has been widely applied in forecasting financial time series data without any parametric assumptions. Myungsook [15] has investigated the effectiveness of inputs in market prediction. Other techniques involve Hybrid Kohonen Self Organisation Map (SOM)[14], Hybrid Financial Systems [17], GA based Support Vector Machines[12], TSK fuzzy rule based systems[16], PCA-MLP model[8], [21], MLP and RBF model[10], Linear regression and Neural Network model[11] and Theil's Nonparametric model[9].

\section{METHODOLOGY}

\section{A. Multivariate Normality}

Multivariate normality plays a crucial role in analyzing data using Statistical procedures. Many Multivariate normality tests are available to test the Marginal normality in a multivariate data. The Shapiro-Wilk test is used if sample size is less than or equal to 5000, otherwise, the Lilliefors test (Kolmogorov-Smirnov test with estimated parameters) is favored. Mardia's skewness and kurtosis coefficients are computed and tests of significance are performed 
for these coefficients using asymptotic distributions. These tests are generally effective for testing multivariate normality. Henze-Zirkler test statistic is found with the associated $p$-value using the lognormal distribution. Finally, the beta $Q-Q$ plot of scaled squared Mahalanobis distances is plotted following the approach of Gnanadesikan and Kettenring (1972).

The test for multivariate normality proposed by Mardia [6][7] which was used to measure the multivariate skewness and kurtosis and joint Multivariate normality. The Henze-Zirkler test is based on a nonnegative functional $D(. .$.$) that measures the$ distance between two distribution functions and has the property that $D\left(N_{d}\left(0, I_{d}\right), Q\right)=0$ if and only if $Q=N_{d}\left(0, l_{d}\right)$ where $N_{d}(u, \Sigma d)$ is a d-dimensional normal distribution. The distance measure $D(. .$.$) can$ be written as

$$
\left.D_{\beta}(P, Q)=\int_{R^{d}}|\hat{P}(t)-\hat{Q}(t)|^{2} \varphi_{S}(t) d t\right\}
$$

where $\hat{P}(t)$ and $\hat{Q}(t)$ are the Fourier transforms of $P$ and $Q$, and $\varphi_{\beta}(t)$ is a weight or a kernel function. The density of the normal distribution $N_{d}\left(0 \beta^{2} I_{d}\right)$ is used as

$$
\varphi_{\beta}(t)=\left(2 \pi \beta^{2}\right)^{\frac{-d}{2}} \exp \left(\frac{-\mid t^{2}}{2 \beta^{2}}\right), t \in R^{d}
$$

where $|t|=(t t)^{0.5}$. The parameter depends on $n$ as

$$
\beta_{n}(n)=\frac{1}{\sqrt{2}}\left(\frac{2 d+1}{4}\right)^{\frac{1}{(d+4)} \frac{1}{n(d+4)}}
$$

The test statistic computed is called $T_{\beta}$ and is approximately distributed as a log normal. The log normal distribution is used to compute the null hypothesis probability.

\section{B. Principal component analysis}

Principal component analysis (PCA) technique consists in rewriting the coordinates in a data set in other coordinates system which will be more convenient for analysis. This new coordinates are represented on orthogonal axis, being obtained in decreasing variance order. The total amount of principal components is equal to the amount of original variables and presents the same statistical information. The PCA is defined as follows:

Let $X=\left(x_{1}, x_{2}, \ldots\right.$ be a $p$ dimensional random variable. The principal component of is $y_{j}=e_{j}^{\prime}=e_{1 j} x_{1}+e_{2 j} x s_{2}+\ldots+e_{p j} x_{p}, \quad i=1,2, \ldots, p$, $e_{j}^{\prime} e_{j}=1$ and it must satisfy the following conditions: The variable is the one whose variance is maximum among all of the variance of $y=$ The variable is not correlative with $y_{1}, y_{2}, \ldots, y_{k}(k=1,2,3, \ldots, p)$

\section{Partial Least Square Regression}

Partial Least Square Regression generalizes and combines the features of principal component analysis and multiple regressions which is obviously related to canonical correlation and to multiple factor analysis. The main originality of PLS regression is to preserve the asymmetry of the relationship between predictors and dependent variables, whereas these other techniques treat them symmetrically. In partial least squares regression, prediction functions are represented by factors extracted from the $Y X X Y$ matrix. The number of such prediction functions that can be extracted typically will exceed the maximum of the number of $Y$ and $X$ variables.

Furthermore, partial least squares regression can be used as an exploratory analysis tool to select suitable predictor variables and to identify outliers before classical linear regression. In PLS the factors to predict the responses in the population are achieved indirectly by extracting latent variables and from sampled factors and responses respectively. The extracted factors are used to predict the $Y$ scores and then the predicted $Y$-scores are used to construct predictions for the responses. In PLS the $X$ - and $Y$-scores are chosen so that the relationship between successive pairs of scores is as strong as possible. In principle, this is like a robust form of redundancy analysis, seeking Directions in the factor space that are associated with high variation in the responses but biasing them toward directions that are accurately predicted.

\section{Multilayer Perceptron}

Neural Networks [NN] have been used in function estimation such as stock price prediction, option price modeling, portfolio optimization and currency exchange rate estimation (Steiner and Wittkemper [13]; Yao and Tan [5]; Galindo [3]; Leigh et al. [20]; Hutchinson et al. 
[4]; Trafalis et al. [9]. NN is a learning machine that is designed to model the way in which the brain performs the particular tasks. The multi-layer perceptron (MLP) is the most widely used type of $\mathrm{NN}$ for function approximation. Input quantities are processed through successive layers of "neurons". Each neuron of a layer other than the input layer computes first a linear combination of the outputs of the neurons of the previous layer, plus a bias. The coefficients of the linear combinations plus the biases are called the weights. Neurons in the hidden layer then compute a non-linear function of their input. The two main activation functions used in current applications are hyperbolic tangent and sigmoid in which hyperbolic tangent ranges from -1 to 1 , and the latter is equivalent in shape but ranges from 0 to 1 .

Multilayer Layer Perceptron has rescaling option which is done to improve the network training. There are three rescaling options: standardization, normalization, and adjusted normalization. All rescaling is performed based on the training data, even if a testing or holdout sample is defined. The units in the output layer can use any one of the following activation function - Identity, Sigmoid, Softmax or Hyperbolic Tangent. Sum of square error and the relative error measures are used to find the best neural network model. Standardized Error Measures used for comparing the ability of the models are

$$
M A P E=\frac{1}{n} \sum_{t=1}^{n} \frac{\left|A_{t}-P_{t}\right|}{A_{t}}
$$

where is the actual value and is the predicted value.

$$
R M S E=\sqrt{\frac{1}{n} \Sigma\left(A_{t}-P_{t}\right)^{2}}
$$

\section{RESULTS AND FINDINGS}

To predict the daily closing prices the variables daily opening, high price, low price, volume of transaction, adjacent closing, stochastic oscillator, Rate of Convergence, momentum, moving averages, disparity and price oscillator are considered.

\section{A. Multivariate Normality}

The marginal normality of the variables is tested using Shapiro Wilks test. Form the Table 1, the p-value of all the variables are less than 0.01 , which implies that the variables are not normally distributed. The joint normality is checked by calculating Mardia's multivariate skewness, multivariate kurtosis and Henze-Zirker test values. In all three evaluations (Table 2), $p$-value is less than 0.01 hence confirming that the multivariate normality is not met. Figure 1 gives the $Q-Q$ plot .Since the assumption of normality is not met nonparametric models can only be used to predict the closing prices.

\section{Beta $Q-Q$ plot}

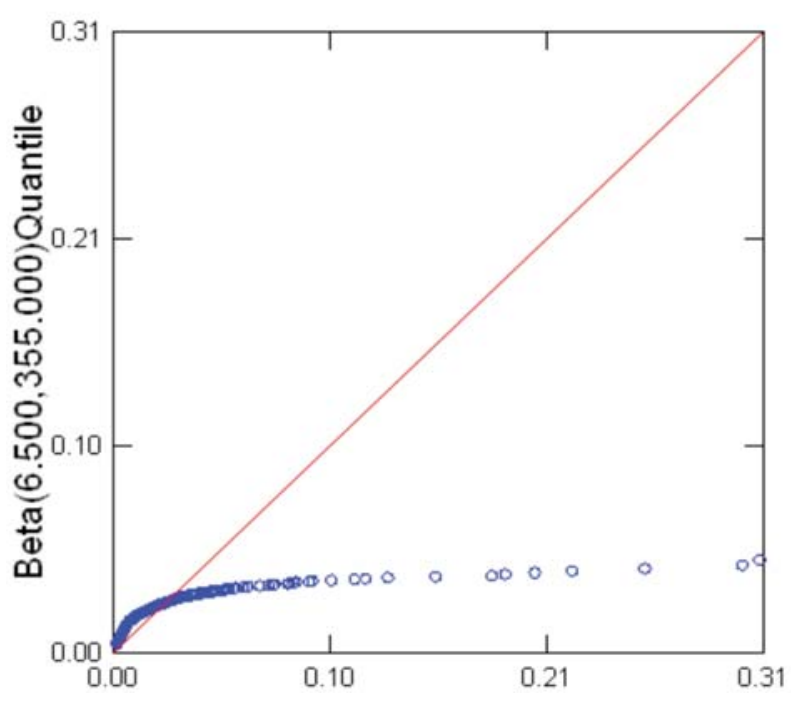

Fig. 1. Scaled Mahalanobis Distances

Table 1. Testing Marginal Normality

\begin{tabular}{|c|c|c|}
\hline Variables & Test Statistic & p-value \\
\hline Open & 0.921 & 0.000 \\
\hline High & 0.920 & 0.000 \\
\hline Low & 0.922 & 0.000 \\
\hline Volume & 0.699 & 0.000 \\
\hline Adj_Close & 0.920 & 0.000 \\
\hline SO & 0.895 & 0.000 \\
\hline ROC & 0.945 & 0.000 \\
\hline Momentum & 0.960 & 0.000 \\
\hline MA5 & 0.917 & 0.000 \\
\hline MA7 & 0.916 & 0.000 \\
\hline MA10 & 0.914 & 0.000 \\
\hline Disparty & 0.942 & 0.000 \\
\hline Price oscillator & 0.941 & 0.000 \\
\hline
\end{tabular}


Table 2. Mardia and Henze-Zirker Test

\begin{tabular}{|c|c|c|c|}
\hline Test & Coefficients & Test Statistic & p-value \\
\hline Mardia Skewness & 164.399 & 19931.441 & 0.000 \\
\hline Mardia Kurtosis & 568.663 & 254.558 & 0.000 \\
\hline Henze-Zirkler & & 5.567 & 0.000 \\
\hline
\end{tabular}

\section{B. Principal Component Analysis}

The predictors are found to be highly collinear in nature. This could be overcome by using the multivariate techniques like Principal component Analysis, Ridge Regression, Partial least square regression and so on. To determine the number of components to be extracted from the variables taken, Scree plot is plotted. From Fig 2, two principal components accounts for maximum variability of the variables. Thus two principal components are extracted from it. Table 3 displays the coefficients of the variables.

Table 3. Coefficient of Variables

\begin{tabular}{|c|c|c|}
\hline Variables & PCA1 & PCA2 \\
\hline Open & 0.723 & 0.689 \\
\hline High & 0.722 & 0.691 \\
\hline Low & 0.731 & 0.681 \\
\hline Volume & -0.993 & 0.122 \\
\hline Adj_Closing & -0.728 & 0.684 \\
\hline Stochastic Oscillator & -0.019 & -0.041 \\
\hline Rate of Convergence & -0.032 & -0.014 \\
\hline Momentum & 0.032 & 0.063 \\
\hline MA5 & 0.027 & 0.685 \\
\hline MA7 & 0.728 & 0.685 \\
\hline MA10 & 0.727 & 0.684 \\
\hline Disparty & 0.49 & 0.024 \\
\hline Price Oscillator & 0.075 & 0.085 \\
\hline
\end{tabular}

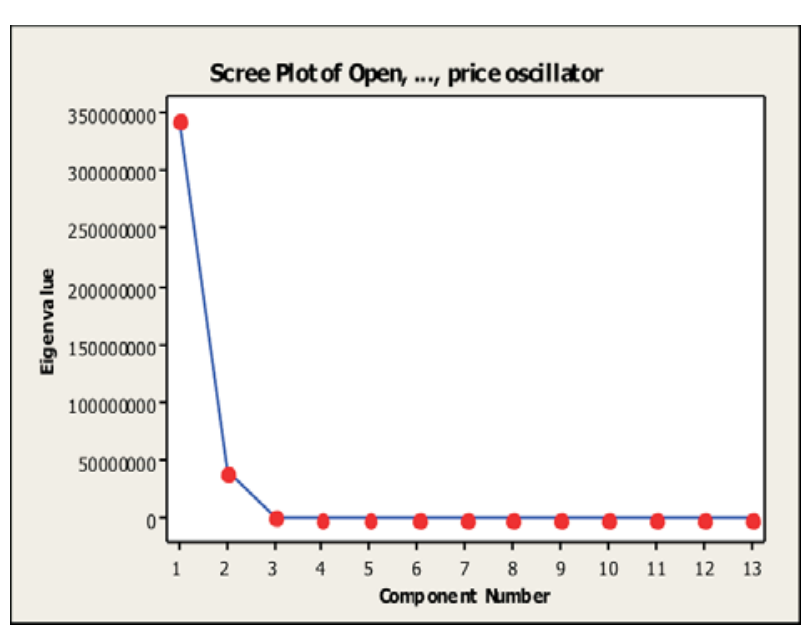

Fig. 2. Scree Plot

\section{Partial Least Square Regression}

Only when seven factors are extracted from the thirteen predictors it accounts for $99.97 \%$ of the total variation (Table 6). The estimates of the regression coefficient are given in Table 5 .

Table 4. Analysis of Variance for Closing Prices

\begin{tabular}{|c|c|c|c|c|c|}
\hline Source & $\begin{array}{c}\text { Sum of } \\
\text { Squares }\end{array}$ & Df & $\begin{array}{c}\text { Mean } \\
\text { Square }\end{array}$ & F & Sig. \\
\hline Reg. & $7.728 \mathrm{E}+09$ & 7 & $1.104 \mathrm{E}+009$ & 748078.6 & 0.00 \\
\hline Error & 1058127.34 & 717 & 1475.770 & & \\
\hline
\end{tabular}

Table 5. Estimate of Regression Coefficient

\begin{tabular}{|c|c|c|}
\hline & Estimate & Standard Error \\
\hline Constant & -3703.264 & 472.053 \\
\hline OPEN & 0.128 & 0.003 \\
\hline HIGH & 0.140 & 0.002 \\
\hline LOW & 0.146 & 0.002 \\
\hline VOLUME & 0.000 & 0.000 \\
\hline ADJ_CLOSE & 0.152 & 0.002 \\
\hline SO & 174.192 & 7.208 \\
\hline ROC & -3386.024 & 475.739 \\
\hline MOMENTUM & 0.214 & 0.031 \\
\hline MA5 & 0.146 & 0.001 \\
\hline MA7 & 0.144 & 0.001 \\
\hline MA10 & 0.143 & 0.001 \\
\hline DISPARTY & 7021.329 & 328.459 \\
\hline PRICE_OSCILLATOR & 2386.821 & 201.388 \\
\hline
\end{tabular}

Table 6. Percent Variation Explained by Factors for Predictors and Responses

\begin{tabular}{|c|c|c|c|c|}
\hline \multirow{2}{*}{ Factors } & \multicolumn{2}{|c|}{$\begin{array}{c}\text { Variation Explained for } \\
\text { Predictor(s) }\end{array}$} & \multicolumn{2}{c|}{$\begin{array}{c}\text { Variation Explained for } \\
\text { Response(s) }\end{array}$} \\
\cline { 2 - 5 } & Percentage & $\begin{array}{c}\text { Cum. } \\
\text { Percentage }\end{array}$ & Percentage & $\begin{array}{c}\text { Cum. } \\
\text { Percentage }\end{array}$ \\
\hline 1 & 57.332 & 57.332 & 99.492 & 99.492 \\
\hline 2 & 6.499 & 63.831 & 0.437 & 99.930 \\
\hline 3 & 14.085 & 77.916 & 0.043 & 99.973 \\
\hline 4 & 12.483 & 90.399 & 0.010 & 99.983 \\
\hline 5 & 5.506 & 95.904 & 0.001 & 99.984 \\
\hline 6 & 3.807 & 99.712 & 0.001 & 99.985 \\
\hline 7 & 0.260 & 99.971 & 0.002 & 99.986 \\
\hline
\end{tabular}




\section{Score Plots}
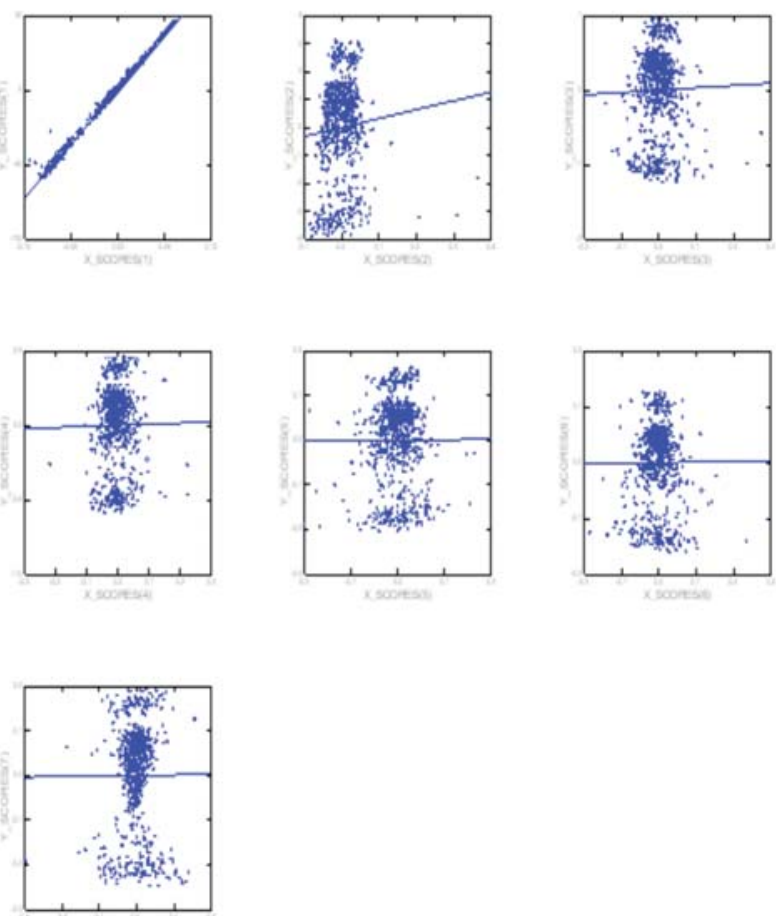

Fig. 3. Score Plot

The value of the estimates and the ANOVA table for the PLS model are given in Tables 5 and 4 respectively. The Score plot in given in Figure 3.

\section{Multilayer Perceptron}

Taking the principal components as covariates and the closing prices as the dependent variable different networks are tried by rescaling the covariates and the dependent variables to standardize, normalize and adjusted normalize.

Table 7. Parameter Estimates

\begin{tabular}{|cc|c|c|c|}
\hline \multirow{2}{*}{ Predictor } & \multicolumn{3}{|c|}{ Predicted } \\
\cline { 3 - 5 } & & \multicolumn{2}{|c|}{ Hidden Layer 1 } & $\begin{array}{c}\text { Output } \\
\text { Layer }\end{array}$ \\
\cline { 3 - 5 } & & $\mathbf{H}(1: 1)$ & $\mathbf{H}(1: 2)$ & Close \\
\hline \multirow{2}{*}{ Input } & (Bias) & .298 & .047 & \\
Layer & pca1 & -.402 & -.032 & \\
& pca2 & -.481 & -.221 & \\
Hidden & (Bias) & & & .542 \\
Layer 1 & $\mathrm{H}(1: 1)$ & & & -.551 \\
& $\mathrm{H}(1: 2)$ & & & -.850 \\
\hline
\end{tabular}

The activation function of the output and the hidden layers are also changed (sigmoid, hyperbolic tangent and identity). The sum of square error and the relative errors are least only when the dependent variables are normalized and the covariates are adjusted normalized with the activation function of the hidden layer is hyperbolic tangent and that of the output layer is identity.

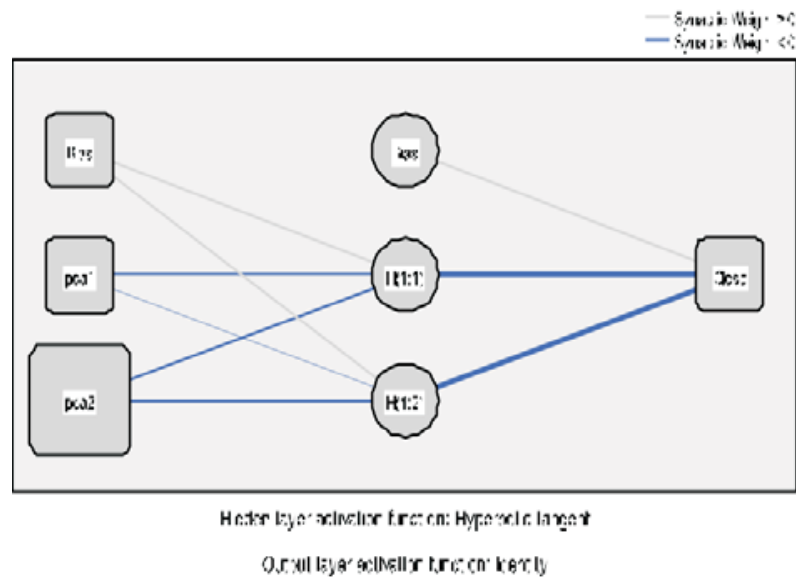

Fig. 4. Network Diagram

Network diagram is given in Figure 4. Root Mean Square Error and the Mean Average Percentage Error values are calculated for all the models proposed. The values are given in Table 8 . The last fifty predicted values of all models with the actual closing prices in given in Figure 5.

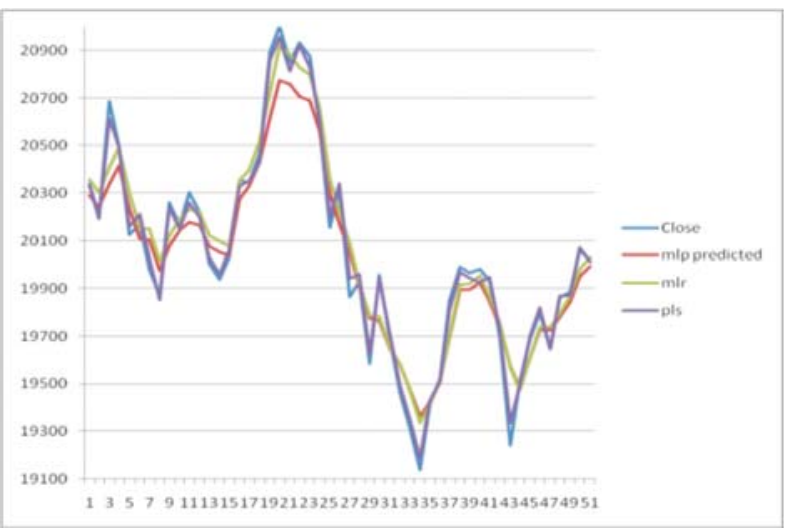

Fig. 5. Actual Vs Predicted Values 
Table 8. Error Measure

\begin{tabular}{|c|c|c|}
\hline Model & RMSE & MAPE \\
\hline MLR & 141.615 & 0.007552 \\
\hline PLS & 38.22171 & 0.001956 \\
\hline MLP & 158.1483 & 0.008583 \\
\hline
\end{tabular}

\section{CONCLUSION}

Comparing the MAPE values, MLP model predicting the daily closing prices with minimum MAPE value but with RMSE we can say that PLS regression surpassed MLP. Hence this model using 7 factors extracted from it is the best model in predicting highly volatile closing prices.

\section{REFERENCES}

[1] Cox D.R. and Small N. J. H., "Testing Multivariate Normality", Biometrika, Volume 65, pp. 263-272.

[2] Cox D.R.and Wermuth, "Tests of linearity, Multivariate Normality and the adequacy of linear scores", Applied Statistics, Volume 43, pp. 347-355.

[3] Galindo J., 1998 " A framework for comparative analysis of statistical and machine learning methods: An application to the black scholes option pricing equations", Techincal report Banco de Mexico, Mexico, DF,04930,.

[4] Hutchinson J. M., Loaw and Poggio T., 1994, "A nonparametic approach to pricing and hedging derivative securities via learning networks",Journal of Finance, Volume 49, pp. 851-889.

[5] Yao J. and Tan C. L., 2000, "A case study on neural networks to perform technical forecasting of forex", Neurocomputing, Volume 34, pp. 79-98.

[6] Mardia K.V., 1970, "Measures of multivariate Skewness and Kurtosis with applications", Biometrika, Volume 57, pp. 519-530.

[7] Mardia K.V., 1980 "Tests of univariate and Multivariate Normality", In Krishnaiah P.R. (ed.), Hand book of Statistics, Volume 1, Chapter 9, Amsterdam, North Holland,.

[8] Lean Yu, Shouyang Wang and Kin Keung Lai,2005, "Mining Stock Market Tendency Using GA Based
Support Vector Machines", X. Dengan Y. Ye (eds): wine LNCS, 3828, Springer Verlag Heidelberg 2005, pp 336-345.

[9] Steiner M. and Wittemper H., 1997, "Portfolio optimization with a neural Network implementation of the coherent market hypothesis", European Jouranl of Operations Research, Volume 100, pp.27-40.

[10] Mark O.Afolabi and Olatoyosi Olider, 2007 "Predicting Stock Prices Using a Hybrid Kohonen Self Organization Map", Proceeding of the $40^{\text {th }}$ Hawaii International Conference on System Science,.

[11] Samreen Fatima, and Ghulam Hussian, 2008, "Statistical models of KSE 100 index using Hybrid Financial Systems", Neuro computing. Elsevier Science Publishers B. V. Volume 71, Issue 13-17, pp 2742-2746.

[12] Trafalis T. B., Ince H. and Mishina T., 2003 "Support Vector Regression in option pricing", Proceedings of Conference on Computational Intelligence and Financial Engineering, Hong Kong.

[13] Leigh W., Paz M. and Purvis R., 2002, "An analysis of a hybrid neural network and pattern recognition techniques for predicting shork-term increases in the nyse", Internation journal of Management Sciences, Volume 30, pp.69-76.

[14] Xiaoping Yang, 2005, "Prediction of Stock Prices Based on PCA and BP Neural Networks", Chinese Business Review, vol. 4, No. 5, S. No. 23, pp 64-68.

[15] Zhang Yudong and Wu Lenan, 2009, "Stock market prediction of S\&P 500 via combination of improved BCO approach and BP neural network", Pergamon Press, Inc. Expert Systems with Applications Volume 36, Issue 5, pp 8849-8854.

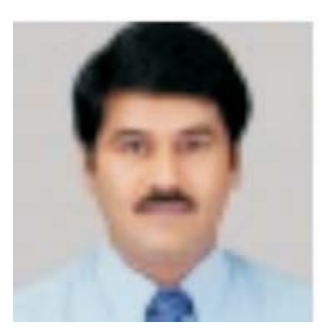

S.Meenakshi Sundaram is working in Sathyabama University, Chennai. He has a teaching experience of 11 years. His fields of interest are Non parametric models, Multivariate statistical methods, statistical inference, Time Series Analysis and Soft Computing. 